\title{
Current fluconazole dosing regimens in critically ill adults result in under-exposure during early therapy
}

\author{
Indy Sandaradura ${ }^{1}$, Deborah Marriott ${ }^{2}$, Richard Day ${ }^{3}$, Ross Norris $^{4}$, Edna Pang ${ }^{4}$, Sophie \\ Stocker $^{5}$, and Stephanie Reuter Lange ${ }^{6}$ \\ ${ }^{1}$ Westmead Hospital \\ ${ }^{2}$ University of New South Wales \\ ${ }^{3}$ St Vincent's Hopsital, Australia \\ ${ }^{4}$ St Vincent's Hospital \\ ${ }^{5}$ University of Sydney \\ ${ }^{6}$ University of South Australia
}

August 17, 2020

\begin{abstract}
Aim: To evaluate current fluconazole dosing regimens against established pharmacodynamic targets in critically ill adults. Methods: Data from critically ill adults treated with fluconazole $(\mathrm{n}=30)$ were used to develop a population pharmacokinetic model. Probability of target attainment (PTA) (fAUC24/MIC >100) was determined from simulations for four previously proposed dosing regimens; (i) $400 \mathrm{mg}$ once daily (ii) a $800 \mathrm{mg}$ loading dose followed by $400 \mathrm{mg}$ once daily, (iii) $400 \mathrm{mg}$ twice daily and (iv) a $12 \mathrm{mg} / \mathrm{kg}$ loading dose followed by $6 \mathrm{mg} / \mathrm{kg}$ once daily. The effect of body weight (40, 70, 120 kg) and renal function (continuous renal replacement therapy (CRRT), 20, 60, 120, $180 \mathrm{~mL} / \mathrm{min}$ glomerular filtration rates) on PTA was assessed. Results: Early (0-48 h) fluconazole target attainment for patients with Minimum Inhibitory Concentrations (MIC) of $2 \mathrm{mg} / \mathrm{L}$ was highly variable. PTA was highest with an $800 \mathrm{mg}$ loading dose for underweight (40 kg) patients and with a $12 \mathrm{mg} / \mathrm{kg}$ loading dose for the remainder. End-of-treatment PTA was highest with $400 \mathrm{mg}$ twice daily maintenance dosing for patients who were under- or normal- weight and $6 \mathrm{mg} / \mathrm{kg}$ maintenance dosing for overweight $(120 \mathrm{~kg})$ patients. None of the fluconazole regimens reliably attained early targets for MICs of [?] $4 \mathrm{mg} / \mathrm{L}$. Conclusion: Current fluconazole dosing regimens do not achieve adequate early target attainment in critically ill adults, particularly in those who are overweight, have higher renal function or are undergoing CRRT. Current fluconazole dosing strategies are generally inadequate to treat organisms with an MIC of [?] $4 \mathrm{mg} / \mathrm{L}$.
\end{abstract}

Article Title: Current fluconazole dosing regimens in critically ill adults result in under-exposure during early therapy

\section{Short Title: Fluconazole dosing in critically ill adults}

Indy SANDARADURA ${ }^{1,2,3,4} *$, Debbie MARRIOTT ${ }^{2,3}$, Richard O DAY ${ }^{3,5}$, Ross L G NORRIS ${ }^{5,6,7}$ Edna PANG $^{5}$, Sophie STOCKER ${ }^{3,5}$ and Stephanie E REUTER ${ }^{8}$

${ }^{1}$ Centre for Infectious Diseases and Microbiology. Westmead Hospital, Sydney, NSW, AUSTRALIA

2 Department of Microbiology, St Vincent's Hospital, Sydney, NSW, AUSTRALIA

${ }^{3}$ St Vincent's Clinical School, University of New South Wales, Sydney, NSW, AUSTRALIA

${ }^{4}$ School of Medicine, University of Sydney, NSW. AUSTRALIA 
${ }^{5}$ Clinical Pharmacology \& Toxicology, St Vincent's Hospital, Sydney, NSW, AUSTRALIA

${ }^{6}$ Discipline of Clinical Pharmacology, School of Medicine \& Public Health, University of Newcastle, Newcastle, NSW, Australia.

${ }^{7}$ Hunter Medical Research Institute, Kookaburra Circuit, New Lambton Heights, NSW, Australia ${ }^{8}$ UniSA Clinical \& Health Sciences, University of South Australia, Adelaide, SA, AUSTRALIA

*Corresponding author. Email indy.sandaradura@health.nsw.gov.au Telephone +61288906012 Fax +61 2 98915317

Principal Investigator statement: The authors confirm that the Principal Investigator for this paper is Indy Sandaradura and that he had direct clinical responsibility for patients.

Word count: 3437 (excluding abstract, references, figures and supplementary material)

Word count (Abstract): 247

Tables: 2

Figures: 2

References: 32

Electronic supplementary material: 1 table, 4 figures and 1 appendix

Key words: fluconazole, population pharmacokinetics, target attainment, critical illness

\title{
What is already known about this subject:
}

- A free fluconazole 24 hour area-under-the-curve to minimum inhibitory concentration $\left(\mathrm{fAUC}_{24} / \mathrm{MIC}\right)$ ratio of [?]100 is associated with a high clinical cure rate.

- Fluconazole pharmacokinetics in the critically ill demonstrate significant interpatient variability, particularly in patients undergoing continuous renal replacement therapy (CRRT) and obese patients.

\section{What this study adds:}

- Early (0-48h) pharmacodynamic target attainment with current fluconazole dosing regimens in critically ill patients is poor, particularly in patients who are overweight, have higher renal function or are undergoing dialysis.

- In general, organisms with an MIC of [?]4 $\mathrm{mg} / \mathrm{L}$ are not adequately treated with fluconazole using current dosing strategies.

\begin{abstract}
:
Aim: To evaluate current fluconazole dosing regimens against established pharmacodynamic targets in critically ill adults.

Methods: Data from critically ill adults treated with fluconazole $(n=30)$ were used to develop a population pharmacokinetic model. Probability of target attainment (PTA) $\left(\mathrm{fAUC}_{24} / \mathrm{MIC}>100\right)$ was determined from simulations for four previously proposed dosing regimens; (i) $400 \mathrm{mg}$ once daily (ii) a $800 \mathrm{mg}$ loading dose followed by $400 \mathrm{mg}$ once daily, (iii) $400 \mathrm{mg}$ twice daily and (iv) a $12 \mathrm{mg} / \mathrm{kg}$ loading dose followed by $6 \mathrm{mg} / \mathrm{kg}$ once daily. The effect of body weight $(40,70,120 \mathrm{~kg}$ ) and renal function (continuous renal replacement therapy (CRRT), 20, 60, 120, $180 \mathrm{~mL} /$ min glomerular filtration rates) on PTA was assessed.

Results: Early $(0-48 \mathrm{~h})$ fluconazole target attainment for patients with Minimum Inhibitory Concentrations (MIC) of $2 \mathrm{mg} / \mathrm{L}$ was highly variable. PTA was highest with an $800 \mathrm{mg}$ loading dose for underweight (40 kg) patients and with a $12 \mathrm{mg} / \mathrm{kg}$ loading dose for the remainder. End-of-treatment PTA was highest with 400
\end{abstract}


mg twice daily maintenance dosing for patients who were under- or normal- weight and $6 \mathrm{mg} / \mathrm{kg}$ maintenance dosing for overweight $(120 \mathrm{~kg})$ patients. None of the fluconazole regimens reliably attained early targets for MICs of [?] $4 \mathrm{mg} / \mathrm{L}$.

Conclusion: Current fluconazole dosing regimens do not achieve adequate early target attainment in critically ill adults, particularly in those who are overweight, have higher renal function or are undergoing CRRT. Current fluconazole dosing strategies are generally inadequate to treat organisms with an MIC of [?]4 mg/L.

\section{Introduction}

Bloodstream infections with Candida spp. are associated with approximately $28 \%$ crude and $11 \%$ attributable mortalities. ${ }^{1}$ In the critically ill, Candida spp. infections are reported to have crude and attributable mortalities of up to 60 and $40 \%$, respectively. ${ }^{2}$ Initiation of adequate antifungal therapy within $48 \mathrm{~h}$ in critically ill patients with candidemia has been demonstrated to lower mortality, especially when combined with other therapeutic measures such as removal of central venous catheters. ${ }^{3}$ Conversely, inadequate dosing of antifungal agents may contribute to treatment failure and increased mortality. ${ }^{4,5}$ The highly variable drug pharmacokinetics $(\mathrm{PK})$ reported in critically ill patients attributed to changes in physiology and the use of extracorporeal devices such as renal replacement therapy ${ }^{6}$ results in inadequate exposure from standard doses of antifungal agents.

Since approval by the FDA and European drug licensing authorities in 1990, fluconazole has been the antifungal agent of choice for fungal infections caused by Candida spp. Fluconazole exhibits linear PK in the recommended dose range and demonstrates excellent distribution into various tissues and body fluids. ${ }^{7-9}$ The free drug 24 hour area under the curve to minimum inhibitory concentration ratio $\left(\mathrm{fAUC}_{24} / \mathrm{MIC}\right)$ is the pharmacodynamic (PD) parameter which best correlates with clinical outcome with a ratio of [?]100 being associated with a high clinical cure rate. ${ }^{10}$ The fluconazole product information recommends maintenance doses of 50 to $400 \mathrm{mg}$ per day and consideration of a loading dose of double the maintenance dose to achieve steady state by 48 h. ${ }^{11}$ By contrast, treatment guidelines for severe Candida spp. infections recommend loading doses of $800 \mathrm{mg}$ and maintenance doses of $400 \mathrm{mg}$ per day. ${ }^{12}$ Weight based dosing $(12 \mathrm{mg} / \mathrm{kg}$ loading; $6 \mathrm{mg} / \mathrm{kg}$ per day maintenance) has also been suggested in some treatment guidelines and may be required to ensure adequate exposure particularly in obese patients. ${ }^{13}$ Finally, renal failure and the use of renal replacement therapies are common in critically ill patients. Fluconazole doses of $800 \mathrm{mg}$ per day for patients receiving continuous renal replacement therapy (CRRT) have been suggested. ${ }^{14-17}$ Generally, the recommended treatment duration for a critically ill patient with invasive candidiasis is 14 days. ${ }^{12}$

The efficacy of fluconazole has been questioned after a patient-level quantitative review of randomized trials investigating treatment strategies in patients with bloodstream infections. ${ }^{18}$ Patients with Candida albicans infection treated with echinocandins, on multivariate analysis, had much lower mortality (OR $0.55,95 \%$ CI $0.32-0.95, \mathrm{p}=0.03$, ) than those treated with other agents to which these organisms were susceptible including fluconazole. In one of the included studies $44 \%$ of patients treated with fluconazole were critically ill and had a higher chance of death (OR 3.8, 95\% CI 1.5-9.6, $\mathrm{p}=0.006) .{ }^{19}$ Although most of the other studies included in this review did not report patient status, given what is known about the epidemiology of candidaemia it is likely that many patients were critically ill. Therefore, these findings may potentially be attributable to the favourable PK profile of echinocandins in critical illness ${ }^{20}$ and raise the possibility that current fluconazole dosing regimens may be suboptimal for these patients. Whilst there has been examination of fluconazole pharmacokinetics in subsets of the critically ill population such as those with renal failure ${ }^{21}$ and those who are obese, ${ }^{22}$ to date no study has comprehensively assessed fluconazole exposure across a heterogenous critically ill population over the entire treatment course.

The objective of this study was to characterise the population PK of fluconazole during the first 14 days of treatment in a diverse but representative cohort of adult patients with critical illness. Further, the product information standard and 3 additional, previously proposed dosing regimens were evaluated against established PD targets across a range of minimum inhibitory concentrations (MICs). 


\section{Patients and methods}

Patients and data collection

Clinical data, including demographics (age, gender, and weight), routine clinical biochemistry data such as serum creatinine (Scr) and serum albumin (ALB) concentrations, and the use of CRRT and extracorporeal membrane oxygenation (ECMO), were obtained from the medical records of consecutive adult patients who were treated in the intensive care unit (ICU) of a tertiary-level teaching hospital in Sydney, Australia between January 2014 and December 2015. Patients received fluconazole doses chosen by their treating physician. Each fluconazole dose was infused at a rate of $200 \mathrm{mg} / \mathrm{h}$ in all patients. Blood samples were collected throughout the dosing interval as part of routine care. For patients not undergoing CRRT, creatinine clearance $\left(\mathrm{CL}_{\mathrm{Cr}}\right)$ was calculated by the Cockroft-Gault formula using total body weight. ${ }^{23}$

Biological sample analysis

Total fluconazole concentrations were determined by a validated assay in a laboratory accredited by the National Association of Testing Authorities against the ISO 15189 standard. In brief, plasma samples were prepared by liquid/liquid extraction followed by gas chromatography with a flame thermionic detector for quantitation. The lower limit of quantification of fluconazole was $1.0 \mathrm{mg} / \mathrm{L}$, with an accuracy (recovery) of $100.5 \%$ and a coefficient of variation (CV) of $9.0 \%$ at this concentration and $98.7 \%$ accuracy, and CV $5.6 \%$ at $45 \mathrm{mg} / \mathrm{L}$. Other biochemical data, including serum creatinine and albumin were determined by the routine pathology service.

Population PK analysis

The PK model development, evaluation, and validation generally followed recent guidelines for PKPD

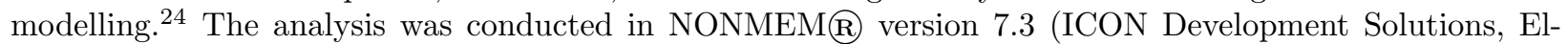
licott City, MD, USA) with a GNU Fortran compiler version 6.1 (GNU Fortran Project, Free Software Foundation, Boston, MA, USA) and PLT Tools interface version 5.4.0 (PLT Soft San Francisco, CA, USA). Model visualisation and evaluation was done in $\mathrm{R}(\mathrm{R}$ ) version 3.4.4 (R Foundation for Statistical Computing, Vienna, Austria). The model incorporated population parameter variability and residual unexplained variability (comprising proportional and/or additive error). Covariance between population parameter variability was assessed. Biologically plausible covariates were tested successively for their influence on PK parameters. Free fluconazole exposure $\left(\mathrm{fAUC}_{24}\right)$ was calculated based on unbound plasma concentrations, assuming protein binding of $12 \% .^{25}$

Model selection was based on a combination of (1) a reduction in objective function value (OFV) of 3.84 units (Chi-square $\left.\left[\chi^{2}\right], \mathrm{p}<0.05\right)$ for nested models with one degree of freedom; (2) graphical goodness-of-fit plots; (3) Akaike's Information theoretic Criterion (AIC); (4) Bayesian Information Criterion (BIC); (5) the biological plausibility of the parameter estimates; and (6) parameter precision. As a visual predictive check (VPC), 1000 simulated datasets and the 5th, 50th and 95th percentiles of model-predicted fluconazole concentrations were plotted against observed data to evaluate the suitability of the final model. For external validation, the final model was also compared with predictions from 2 other published models in critically ill populations. ${ }^{21,26}$ Published, observed concentration-time data ${ }^{21}$ were scanned, extracted using DigitizeIt software (I Bormann, Braunschweig, Germany) and overlaid on the model predictions for equivalent populations.

Dosing simulations

To examine current and proposed fluconazole dosing regimens in patients with critical illness, the final population PK model was used to simulate datasets for 15,000 patients with representative body weights $(40,70,120 \mathrm{~kg})$ and renal function (CRRT, 20, 60, 120, $180 \mathrm{~mL} / \mathrm{min})$. Pharmacodynamic target attainment was defined as $\mathrm{fAUC}_{24} / \mathrm{MIC}>100$. Probability of target attainment (PTA) was determined for four different dosing regimens at $0-24 \mathrm{~h}, 24-48 \mathrm{~h}$ and at $312-336 \mathrm{~h}$. Daily target attainment was also determined for an MIC of $2 \mathrm{mg} / \mathrm{L}$ over the entire treatment course. The dosing regimens examined were (i) $400 \mathrm{mg}$ once daily (ii) an $800 \mathrm{mg}$ loading dose followed by $400 \mathrm{mg}$ once daily, (iii) $400 \mathrm{mg}$ twice daily and (iv) a $12 \mathrm{mg} / \mathrm{kg}$ 
loading dose followed by $6 \mathrm{mg} / \mathrm{kg}$ once daily. A range of MICs from 0.5 to $8 \mathrm{mg} / \mathrm{L}$ were examined, with a focus on the upper limits of the susceptible range as defined by European Committee on Antimicrobial Susceptibility Testing (EUCAST), $2 \mathrm{mg} / \mathrm{L}$ and Clinical and Laboratory Standards Institute (CLSI), $4 \mathrm{mg} / \mathrm{L}$. Organisms with these MICs are deemed by these organisations to be treatable with the standard fluconazole regimen. Simulations were performed using NONMEM. Data processing was conducted using Rß[?]. ${ }^{27}$

\section{Results}

Study population and patient characteristics

A total of 30 critically ill patients were enrolled in the study for which 130 plasma fluconazole concentrations (median 4, range 2-11 observations per patient) were reported and included in the analysis. No samples were excluded from the analysis dataset. The characteristics of the patients are presented in Table 1. Loading doses (800 mg to $1600 \mathrm{mg}$ ) were administered to $63 \%$ (19/30) of patients and fluconazole maintenance doses ranged from $200 \mathrm{mg}$ to $800 \mathrm{mg}$ per day. Albumin concentrations were low $(<36 \mathrm{~g} / \mathrm{L})$ in the majority $(87 \%$ [26/30]) of patients. Reduced renal function $\left(\mathrm{CL}_{\mathrm{Cr}}<90 \mathrm{~mL} / \mathrm{min}\right)$ was documented in $63 \%(19 / 30)$ of patients and 6 of these patients received continuous renal replacement therapy (CRRT). Extracorporeal membrane oxygenation (ECMO) was undertaken in $17 \%(5 / 30)$ of patients.

Population PK model building and evaluation

A one compartment model with first order elimination and a combined error model was found to best describe fluconazole concentration-time data (Table S1). Introduction of covariates into the structural model identified a significant effect of total body weight on volume of distribution $(\mathrm{Vc})$ and creatinine clearance $\left(\mathrm{CL}_{\mathrm{Cr}}\right)$ and the use of CRRT on clearance (CL). No significant relationships were observed between fluconazole PK parameters and gender, age and the use of ECMO. A variance-covariance matrix for the variability in CL and Vd was included in the final model.

The final model control stream is presented in Appendix S1. The diagnostic plots used to confirm the appropriateness of the final model are presented in Figures S1, S2 and S3. Additional model validation comparing predictions from our model to predicted and observed data from 2 other published population PK models ${ }^{21,26}$ for identical test populations demonstrated good agreement. The final population PK model is illustrated in Figure 1 and the final model parameters are summarised in Table 2.

Assessment of dose-exposure relationship

Probability of target attainment (PTA) at (a) $48 \mathrm{~h}$ and (b) end of treatment for the 4 different fluconazole regimens examined for a range $(0.5$ to $8 \mathrm{mg} / \mathrm{L})$ of MICs, stratified by weight and renal function is presented in Figure S4. For simplicity the results for the two $400 \mathrm{mg}$ once daily maintenance regimens are presented together in Figure S4 (b) as their end of treatment PTA is identical. PTA over time for organisms with an MIC of (a) $2 \mathrm{mg} / \mathrm{L}$ and (b) $4 \mathrm{mg} / \mathrm{L}$ for the same fluconazole regimens and patient groups is presented in Figure 2 .

\section{Early (0-48h) target attainment}

MIC $2 \mathrm{mg} / \mathrm{L}$

Early target attainment for an isolate with an MIC of $2 \mathrm{mg} / \mathrm{L}$ in the absence of a loading dose was suboptimal $(<95 \%)$ for most patients except those who were underweight $(40 \mathrm{~kg})$. Target attainment at $0-24 \mathrm{~h}$ was highest (97 - 100\%) with an $800 \mathrm{mg}$ loading dose for underweight patients and with a $12 \mathrm{mg} / \mathrm{kg}$ loading dose for the remainder. Even with a $12 \mathrm{mg} / \mathrm{kg}$ loading dose target attainment decreased as weight and $\mathrm{CL}_{\mathrm{Cr}}$ increased and amongst patients with the highest renal function $\left(\mathrm{CL}_{\mathrm{Cr}} 180 \mathrm{~mL} / \mathrm{min}\right)$, only $86 \%$ of those who weighed $70 \mathrm{~kg}$ and $90 \%$ of those who weighed $120 \mathrm{~kg}$ met targets. Target attainment at $24-48 \mathrm{~h}$ for an isolate with an MIC of $2 \mathrm{mg} / \mathrm{L}$ was highest ([?]98\%) with $400 \mathrm{mg}$ twice daily for patients who were under- or normal- weight. Overweight patients had the highest target attainment ([?]99\%) with $6 \mathrm{mg} / \mathrm{kg}$ maintenance dosing. These findings were replicated in patients on CRRT at 24-48 h. 
With the commonly prescribed guideline dose of $800 \mathrm{mg}$ loading dose followed by $400 \mathrm{mg}$ once daily early target attainment was poor in the most extreme simulated patients $\left(120 \mathrm{~kg}, \mathrm{CL}_{\mathrm{Cr}} 120 \mathrm{~mL} / \mathrm{min}\right.$ and 120 $\mathrm{kg}, \mathrm{CL}_{\mathrm{Cr}} 180 \mathrm{~mL} / \mathrm{min}$ ) where only 79 and $72 \%$ of patients respectively met targets at $24-48 \mathrm{~h}$. With this regimen, it took until $96 \mathrm{~h}$ for patients who were $120 \mathrm{~kg}$ with a $\mathrm{CL}_{\mathrm{Cr}}$ of $120 \mathrm{~mL} / \mathrm{min}$ and $288 \mathrm{~h}$ for patients who were $120 \mathrm{~kg}$ with a $\mathrm{CL}_{\mathrm{Cr}}$ of $180 \mathrm{~mL} / \mathrm{min}$ for $>95 \%$ target attainment. At $24-48 \mathrm{~h}$ target attainment for CRRT patients was variable (17-61\%) after $800 \mathrm{mg}$ loading followed by $400 \mathrm{mg}$ once daily and decreased over time as the impact of the loading dose was lost.

MIC $4 \mathrm{mg} / \mathrm{L}$

For an isolate with an MIC of $4 \mathrm{mg} /$ the probability of early target attainment was low for all the tested fluconazole regimens. At 0-24 h target attainment was highest (44-77\%) with an $800 \mathrm{mg}$ loading dose for underweight patients and with a $12 \mathrm{mg} / \mathrm{kg}$ loading dose for the remainder (11-31\%). Target attainment at 24-48 $\mathrm{h}$ for an isolate with an MIC of $4 \mathrm{mg} / \mathrm{L}$ was highest (56-99\%) with $400 \mathrm{mg}$ twice daily for patients who were under- or normal- weight. Overweight patients had the highest target attainment (58-84\%) with $6 \mathrm{mg} / \mathrm{kg}$ maintenance dosing. Target attainment decreased with increasing $\mathrm{CL}_{\mathrm{Cr}}$.

With the commonly prescribed guideline dose of $800 \mathrm{mg}$ loading dose followed by $400 \mathrm{mg}$ once daily early target attainment was negligible in the most extreme simulated patients $\left(120 \mathrm{~kg}, \mathrm{CL}_{\mathrm{Cr}} 120 \mathrm{~mL} / \mathrm{min}\right.$ and $120 \mathrm{~kg}, \mathrm{CL}_{\mathrm{Cr}} 180 \mathrm{~mL} / \mathrm{min}$ ) where only 1 and $0 \%$ of patients respectively met targets at $24-48 \mathrm{~h}$. With this regimen $>95 \%$ target attainment was not achieved during the treatment course in these patients.

All the tested fluconazole regimens resulted in $<7 \%$ target attainment in patients on CRRT at 0-48 h.

\section{End of treatment $(336 \mathrm{~h})$ target attainment}

End of treatment target attainment was $100 \%$ for an isolate with an MIC of $2 \mathrm{mg} / \mathrm{L}$ with $400 \mathrm{mg}$ twice daily maintenance dosing for all patient groups. The standard maintenance fluconazole dose of $400 \mathrm{mg}$ also performed well in patients who were not on CRRT with [?]95\% target attainment at the end of treatment. In CRRT patients $400 \mathrm{mg}$ once daily resulted in [?] $24 \%$ target attainment whilst $6 \mathrm{mg} / \mathrm{kg}$ only led to satisfactory target attainment in overweight patients.

For an isolate with an MIC of $4 \mathrm{mg} / \mathrm{L}$ the $400 \mathrm{mg}$ twice daily fluconazole regimen resulted in $>95 \%$ of patients who were not receiving CRRT meeting PD targets. Although this regimen also performed the best in CRRT patients, $<24 \%$ met targets. Whilst the standard maintenance regimen of $400 \mathrm{mg}$ once daily resulted in patients with impaired renal function $\left(\mathrm{CL}_{\mathrm{Cr}} 20 \mathrm{~mL} / \mathrm{min}\right)$ meeting targets, it proved to be suboptimal in those with normal or augmented renal function and those on CRRT.

\section{MIC [?] $1 \mathrm{mg} / \mathrm{L}$ target attainment}

The tested fluconazole regimens were found to result in reliable target attainment for organisms with an MIC of [?] $1 \mathrm{mg} / \mathrm{L}$ in all but the most extreme CRRT patients. Early target attainment for $120 \mathrm{~kg}$ patients receiving $400 \mathrm{mg}$ once daily was $88 \%$ and end of treatment target attainment for $40 \mathrm{~kg}$ patients receiving $6 \mathrm{mg} / \mathrm{kg}$ maintenance dosing was $79 \%$ for isolates with an MIC of $1 \mathrm{mg} / \mathrm{L}$.

\section{Discussion}

To date there has been limited study of the population PK of fluconazole in the critically ill despite the widespread use of the drug in this population to treat serious infections. Significant interpatient variability in fluconazole PK has been demonstrated in intensive care patients $(\mathrm{n}=57),{ }^{26}$ anuric patients undergoing CRRT $(\mathrm{n}=10)^{21}$ and obese intensive care patients $(\mathrm{n}=37)^{22}$. Although each of these studies examined the pharmacokinetics of fluconazole in a subset of critically ill patients the prevalence of altered body weight (both malnourishment and obesity), altered renal function (both augmented and diminished) and the prevalence of renal replacement therapies and their frequent coexistence in intensive care patients means that these studies inadequately describe the PK of fluconazole across the entire critical care population. 
In our study the population PK of intravenous fluconazole in critically ill adult patients was characterised using a one-compartment model. Although Patel et al fitted their data to a 2 compartment model, our model adequately describes their data. Consistent with previous studies, ${ }^{22,26}$ total body weight was a covariate for $\mathrm{Vc}$ and CLcr a covariate for CL. The final parameters in our model for Vc and CL and $\mathrm{CL}_{\mathrm{CRRT}}$ are similar to those reported previously. ${ }^{21,26}$ Consistent with this, the model was able to accurately predict the observed concentrations of fluconazole in intensive care patients and patients on CRRT. This indicates the generalisability and robustness of the model to predict fluconazole drug exposure across a broad range of critically ill patient populations.

Subsequently, the model was used to examine the pharmacodynamic target attainment of previously described dosing regimens. We have demonstrated that pharmacodynamic targets are not consistently achieved at 0-48 $\mathrm{h}$ in overweight patients, those with higher renal function or those undergoing CRRT despite administration of the currently recommended loading dose $(800 \mathrm{mg})$. Patients with these characteristics are commonly treated in the intensive care unit and made up 13/30 (43\%) of our study population. Poor early target attainment with standard fluconazole dosing in a substantial proportion of patients who are critically ill may in part explain the worse outcomes described in these patients. ${ }^{1,2} \mathrm{It}$ is likely that a $12 \mathrm{mg} / \mathrm{kg}$ loading dose would perform better in patients who are not underweight.

We have also demonstrated that whilst $400 \mathrm{mg}$ /day maintenance dosing is sufficient to reliably treat infections caused by organisms with an MIC [?]2 mg/L, higher doses (400 $\mathrm{mg}$ twice daily) are required for patients receiving CRRT. Although other authors have also suggested higher doses during CRRT, ${ }^{14-17}$ existing treatment guidelines ${ }^{12}$ and drug monographs ${ }^{28,29}$ including the fluconazole product information ${ }^{11}$ do not yet make this recommendation. Therefore, most clinicians are unlikely to be aware of the need to administer higher doses of fluconazole in these clinical circumstances.

From our data it is apparent that an $\mathrm{fAUC}_{24} / \mathrm{MIC}$ of $>100$ for organisms with an MIC of $4 \mathrm{mg} / \mathrm{L}$ would not be achieved reliably during the early stages of therapy with any one current or proposed fluconazole dosing regimen in patients with critical illness. Higher maintenance doses (400 $\mathrm{mg}$ twice daily) than those currently in use would also be required for end of treatment target attainment in patients who are not receiving CRRT. Although the use of several different dosing regimens based on weight, renal function and fluconazole MIC may improve target attainment across the population, successful implementation of dosing advice for the variable regimens needed to accommodate the relevant, individual covariates of critically ill patients' will present logistic challenges.

In a recent multicentre Australian study, organisms with MICs of 2 and $4 \mathrm{mg} / \mathrm{L}$ accounted for $12 \%$ and $2 \%$ respectively of all Candida albicans bloodstream infections. ${ }^{30}$ In current laboratory practice these organisms are deemed to be amenable to treatment with the standard fluconazole regimen, indicating to clinicians a high probability of success. Given the significant risk of mortality associated with these infections, clinicians treating critically ill patients need to be mindful of the poor early target attainment in some patients with current and proposed fluconazole dosing regimens.

Several potential limitations of this study must be considered. The first is our choice of fAUC/MIC >100 as the PD threshold predictive of a good outcome. Although this is used by many clinicians and supported by EUCAST, other authors have proposed thresholds ranging from [?]11.5 ${ }^{19}$ to [?]400. ${ }^{31}$ Utilising a higher PD threshold would mean that even fewer patients would attain targets with current dosing regimens, whilst a lower PD threshold would result in most patients being adequately treated. A second limitation is that we estimated free fluconazole concentrations rather than measuring them. Changes in protein binding are known to occur during critical illness. However, it is those drugs which are the most highly bound where free drug concentrations become the most difficult to predict and small absolute changes may result in large proportional changes. ${ }^{32}$ Given the relatively low extent of fluconazole protein binding, the impact on the results is unlikely to be significant. 


\section{Conclusions}

This population pharmacokinetic analysis strongly indicates that fluconazole dosage should be optimized in terms of weight, $\mathrm{CL}_{\mathrm{Cr}}$ and CRRT in critically ill patients in order to ensure pharmacodynamic target attainment for organism MICs of $2 \mathrm{mg} / \mathrm{L}$. Whilst we were able to support the EUCAST clinical breakpoint of $>2 \mathrm{mg} / \mathrm{L}$ when only considering end of treatment target attainment, it was apparent that patients with higher renal function, those undergoing CRRT and those who are overweight require increased doses to attain these same targets early and maximise the chance of achieving a good clinical outcome. Conversely, it is likely that lower doses would be sufficient to attain these targets in underweight patients and those with lower renal function. In the interests of stewardship of existing antimicrobial agents, it is critical to ensure that the use of established drugs such as fluconazole is optimised before relegation in favour of newer (and usually more expensive) options. As no single current or proposed fluconazole dosing regimen resulted in optimal exposure across the studied critically ill population, further clinical studies with optimised dosing of fluconazole using population PK are warranted.

\section{Transparency declarations:}

Ethical approval and consent to participate

The study was approved by the Institutional Human Research Ethics Committee (LNR/14/SVH/148).

Availability of supporting data

The data used for this research are available from the corresponding author on reasonable request and subject to Institutional Ethics Committee guidelines.

Competing interests

None to declare

Funding

This research was supported by an Australian Government Research Training Program (RTP) Scholarship to IS. Work undertaken by S.E.R. is with the financial support of Cancer Council's Beat Cancer Project on behalf of its donors, the State Government through the Department of Health, and the Australian Government through the Medical Research Future Fund.

Authors' contributions

IS designed the study, collected data, conducted pharmacokinetic modelling, performed data analysis and prepared the manuscript. ROD assisted with study design. DM conceived the study. EP and RLGN supervised analysis of plasma concentrations and assisted with data collection. SER supervised pharmacokinetic modelling and data analysis. All of the authors reviewed the manuscript.

Acknowledgements

The preliminary results of this study were presented at the World Conference of Pharmacometrics 2016 (Poster 217).

The authors wish to thank the Department of Intensive Care, St Vincent's Hospital and the TDM laboratory, Sydpath for their assistance with collection and assaying of specimens respectively.

\section{References:}

1. Chen S, Slavin M, Nguyen Q, et al. Active surveillance for candidemia, Australia.Emerg Infect Dis 2006; 12: $1508-16$.

2. Eggimann P, Garbino J, Pittet D. Epidemiology of Candida species infections in critically ill nonimmunosuppressed patients. Lancet Infect Dis 2003;3: 685-702. 
3. Puig-Asensio M, Peman J, Zaragoza R, et al. Impact of therapeutic strategies on the prognosis of candidemia in the ICU. Crit Care Med 2014;42: 1423-32.

4. Labelle AJ, Micek ST, Roubinian N, Kollef MH. Treatment-related risk factors for hospital mortality in Candida bloodstream infections. Crit Care Med 2008;36: 2967-72.

5. Zilberberg MD, Kollef MH, Arnold H, et al. Inappropriate empiric antifungal therapy for candidemia in the ICU and hospital resource utilization: a retrospective cohort study. BMC Infect Dis 2010; 10: 150.

6. Roberts JA, Abdul-Aziz MH, Lipman J, et al. Individualised antibiotic dosing for patients who are critically ill: challenges and potential solutions. Lancet Infect Dis 2014; 14: 498-509.

7. Pappas PG, Kauffman CA, Andes D, et al. Clinical practice guidelines for the management of candidiasis: 2009 update by the Infectious Diseases Society of America. Clin Infect Dis 2009; 48: 503-35.

8. Charlier C, Hart E, Lefort A, et al. Fluconazole for the management of invasive candidiasis: where do we stand after 15 years? J Antimicrob Chemother 2006;57: 384-410.

9. Thaler F, Bernard B, Tod M, et al. Fluconazole penetration in cerebral parenchyma in humans at steady state. Antimicrob Agents Chemother 1995;39: 1154-6.

10. Rodriguez-Tudela JL, Almirante B, Rodriguez-Pardo D, et al. Correlation of the MIC and Dose/MIC Ratio of Fluconazole to the Therapeutic Response of Patients with Mucosal Candidiasis and Candidemia. Antimicrob Agents Chemother 2007; 51: 3599-604.

11. Anon. DIFLUCAN.Product Information $2014 . \quad$ Available at: http://labeling.pfizer.com/ShowLabeling.aspx?id=575. Accessed January 5, 2017.

12. Pappas PG, Kauffman CA, Andes DR, et al. Clinical Practice Guideline for the Management of Candidiasis: 2016 Update by the Infectious Diseases Society of America. Clin Infect Dis 2016; 62: e1-50.

13. Alobaid AS, Wallis SC, Jarrett P, et al. What is the effect of obesity on the population pharmacokinetics of fluconazole in critically ill patients?Antimicrob Agents Chemother 2016. Available at: http://dx.doi.org/10.1128/AAC.01088-16.

14. Bergner R, Hoffmann M, Riedel K-D, et al. Fluconazole dosing in continuous veno-venous haemofiltration (CVVHF): need for a high daily dose of $800 \mathrm{mg}$. Nephrol Dial Transplant 2006; 21: 1019-23.

15. Muhl E, Martens T, Iven H, Rob P, Bruch H-P. Influence of continuous veno-venous haemodiafiltration and continuous veno-venous haemofiltration on the pharmacokinetics of fluconazole. Eur J Clin Pharmacol 2000;56: 671-8.

16. Pittrow L, Penk A. Dosage adjustment of fluconazole during continuous renal replacement therapy (CAVH, CVVH, CAVHD, CVVHD). Mycoses 1999; 42: 17-9.

17. Yagasaki K, Gando S, Matsuda N, et al. Pharmacokinetics and the most suitable dosing regimen of fluconazole in critically ill patients receiving continuous hemodiafiltration. Intensive Care Med 2003; 29: 1844-8.

18. Andes DR, Safdar N, Baddley JW, et al. Impact of treatment strategy on outcomes in patients with candidemia and other forms of invasive candidiasis: a patient-level quantitative review of randomized trials. Clin Infect Dis 2012; 54: 1110-22.

19. Baddley JW, Patel M, Bhavnani SM, Moser SA, Andes DR. Association of fluconazole pharmacodynamics with mortality in patients with candidemia.Antimicrob Agents Chemother 2008; 52: 3022-8.

20. Muilwijk EW, Schouten JA, van Leeuwen HJ, et al. Pharmacokinetics of caspofungin in ICU patients. J Antimicrob Chemother 2014; 69: 3294-9. 
21. Patel K, Roberts JA, Lipman J, Tett SE, Deldot ME, Kirkpatrick CM. Population pharmacokinetics of fluconazole in critically ill patients receiving continuous venovenous hemodiafiltration: using Monte Carlo simulations to predict doses for specified pharmacodynamic targets. Antimicrob Agents Chemother 2011; 55: $5868-73$.

22. Alobaid AS, Wallis SC, Jarrett P, et al. Population Pharmacokinetics of Piperacillin in Nonobese, Obese, and Morbidly Obese Critically Ill Patients.Antimicrob Agents Chemother 2017; 61. Available at: http://dx.doi.org/10.1128/AAC.01276-16.

23. Cockcroft DW, Gault MH. Prediction of creatinine clearance from serum creatinine. Nephron1976; 16: $31-41$.

24. Nguyen THT, Mouksassi M-S, Holford N, et al. Model Evaluation of Continuous Data Pharmacometric Models: Metrics and Graphics. CPT Pharmacometrics Syst Pharmacol 2017; 6: 87-109.

25. Debruyne D, Ryckelynck JP. Clinical pharmacokinetics of fluconazole. Clin Pharmacokinet1993; 24: $10-27$.

26. Aoyama T, Hirata K, Hirata R, et al. Population pharmacokinetics of fluconazole after administration of fosfluconazole and fluconazole in critically ill patients. J Clin Pharm Ther 2012; 37: 356-63.

27. R Development Core Team. R: A language and environment for statistical computing. 2017. Available at: https://www.R-project.org/.

28. Nett J, Andes D. Fluconazole. In: Kucers' The Use of Antibiotics Sixth Edition. CRC Press, 2010; $1806-23$.

29. Anon. Fluconazole: Dosing/Administration. Micromedex 2016. Available at: http://www.micromedexsolutions.com/. Accessed January 5, 2017.

30. van Hal SJ, Chen SC-A, Sorrell TC, Ellis DH, Slavin M, Marriott DM. Support for the EUCAST and revised CLSI fluconazole clinical breakpoints by Sensititre(r) YeastOne(r) for Candida albicans: a prospective observational cohort study. J Antimicrob Chemother 2014; 69: 2210-4.

31. Brosh-Nissimov T, Ben-Ami R. Differential association of fluconazole dose and dose/MIC ratio with mortality in patients with Candida albicans and non-albicans bloodstream infection. Clin Microbiol Infect 2015; 21: 1011-7.

32. Roberts JA, Pea F, Lipman J. The clinical relevance of plasma protein binding changes. Clin Pharmacokinet 2013; 52: 1-8.

Table 1: Clinical data for study subjects at time of first fluconazole concentration measurement ${ }^{\text {a }}$

\begin{tabular}{ll}
\hline Characteristic & Value (range) \\
Age & $55 \mathrm{y}(21-80)$ \\
Male & $18 / 30$ \\
Weight & $80 \mathrm{~kg}(40-115)$ \\
Fluconazole maintenance dose & $400 \mathrm{mg}(200-800)$ \\
Loading dose & $19 / 30$ \\
Serum creatinine & $107 \mathrm{umol} / \mathrm{L}(35-420)$ \\
Creatinine clearance ${ }^{b}$ & $78 \mathrm{~mL} / \mathrm{min}(26-182)$ \\
Albumin & $29 \mathrm{~g} / \mathrm{L}(15-46)$ \\
Continuous renal replacement therapy & $6 / 30$ \\
Extracorporeal membrane oxygenation & $5 / 30$ \\
Continuous renal replacement therapy and Extracorporeal membrane oxygenation & $2 / 30$ \\
Sequential Organ Failure Assessment Score & $7(4-10)$ \\
\hline
\end{tabular}


${ }^{\mathrm{a}} \mathrm{n}=130 .{ }^{\mathrm{b}}$ Non-CRRT patients. Values presented as median (range), except for gender, loading dose, CRRT and ECMO which are presented as fractions.

Table 2: Final population PK model parameter estimates

\begin{tabular}{|c|c|c|c|c|}
\hline Parameter & Estimate & Standard Error & Shrinkage & Eta Bar P \\
\hline \multicolumn{5}{|l|}{ Fixed effects } \\
\hline$C L_{\text {sys }}(L / h)$ & 0.705 & 0.0850 & & \\
\hline$V c(L)$ & 46.3 & 3.51 & & \\
\hline$C L_{C R R T}(L / h)$ & 1.91 & 0.0938 & & \\
\hline$C L_{C r}{ }^{\sim} C L_{\text {sys }}$ & 0.635 & 0.306 & & \\
\hline$W t^{\sim} V d$ & 0.900 & 0.193 & & \\
\hline \multicolumn{5}{|l|}{ Random effects } \\
\hline$B S V C L_{\text {sys }}(\% C V)$ & 39.7 & & $24.7 \%$ & 0.973 \\
\hline$B S V V c(\% C V)$ & 33.0 & & $14.9 \%$ & 0.791 \\
\hline$B S V C L_{C R R T}(\% C V)$ & 12.4 & & $59.4 \%$ & 0.934 \\
\hline$V c-C L_{\text {sys }}($ Correlation $)$ & 0.349 & & & \\
\hline \multicolumn{5}{|l|}{ Residual variability } \\
\hline additive $(\mathrm{mg} / \mathrm{L})$ & 2.25 & & $15.8 \%$ & \\
\hline proportional $(\% C V)$ & 4.58 & & $15.8 \%$ & \\
\hline
\end{tabular}

Figure 1: Final population PK model. $\vartheta_{\mathrm{i}}$ individual parameter; $\vartheta_{\mathrm{p}}$ population parameter; $\eta_{\mathrm{i}}$ random effect of between-subject variability; $\mathrm{CL}_{\text {sys }}$ systemic renal clearance, $\mathrm{CL}_{\mathrm{CRRT}}$ systemic clearance on CRRT, CLCr creatinine clearance; $\mathrm{CLCr} \sim \mathrm{CL}$ effect of creatinine clearance on clearance; $\mathrm{V}_{\mathrm{c}}$ central compartment volume of distribution; WT body weight; $\mathrm{Wt}^{\sim} \mathrm{Vc}$ effect of body weight on volume of distribution

Figure 2: Probability of target attainment for an organism MIC of ${ }^{\mathrm{a}} 2 \mathrm{mg} / \mathrm{L}$ and ${ }^{\mathrm{b}} 4 \mathrm{mg} / \mathrm{L}$ over time of tested fluconazole dosing regimens in critically ill adult patients stratified by weight and renal function. 


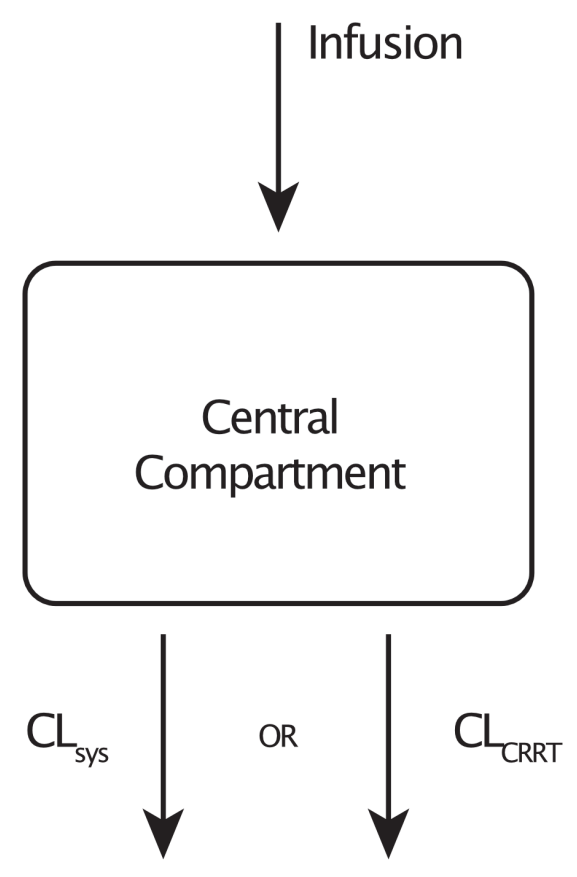

$$
\begin{array}{ll}
\text { IF CRRT }=0 & \theta_{i} C L=\theta_{p} C L_{\text {sys }} \cdot\left(\left(C L_{C r} / 120\right) \wedge \theta_{p}^{C L C r-C L}\right) \cdot \exp \left(\eta_{i}^{C L}\right) \\
I F C R R T=1 & \theta_{i} C L=\theta_{p} C L_{C R R T} \cdot \exp \left(\eta_{i}^{C L C R R T}\right)
\end{array}
$$

$$
\theta_{i} V_{c}=\theta_{p} V_{c} \cdot\left((W T / 70) \wedge \theta_{p}{ }^{W t} v_{c}\right) \cdot \exp \left(\eta_{i}^{V_{c}}\right)
$$

(a) Target attainment over time by regimen for an MIC of $2 \mathrm{mg} / \mathrm{L}$, stratified by weight and renal function

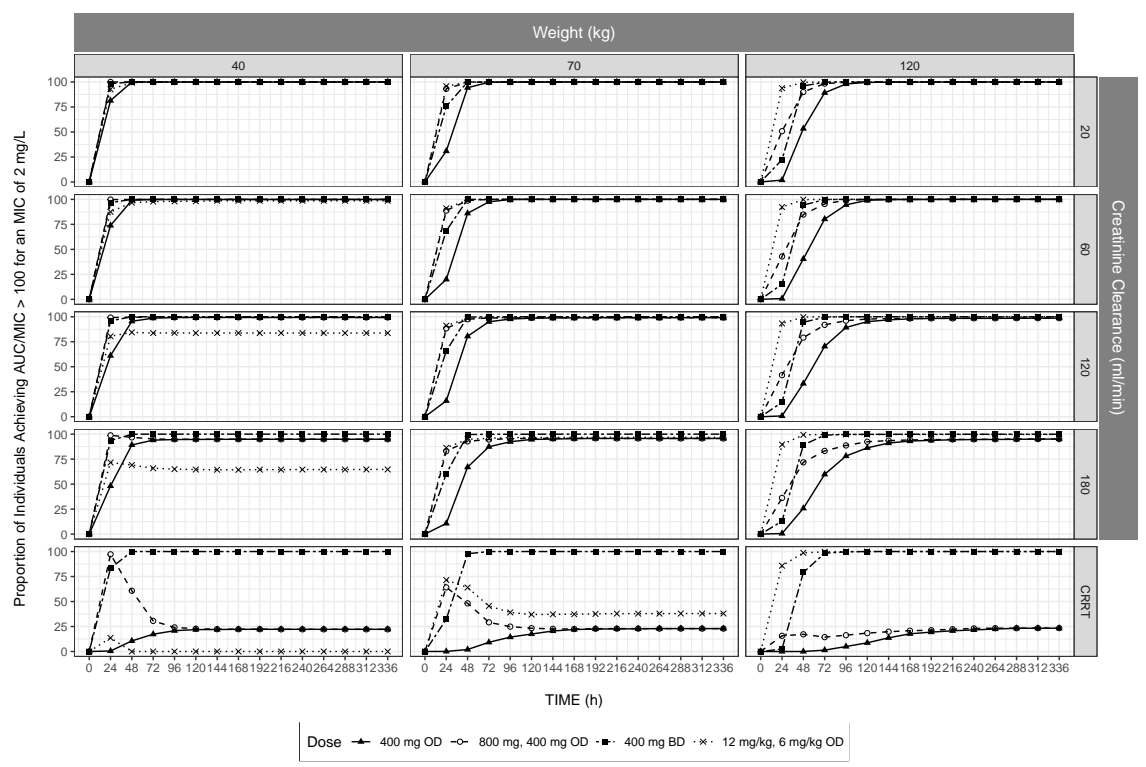


(b) Target attainment over time by regimen for an MIC of $4 \mathrm{mg} / \mathrm{L}$, stratified by weight and renal function

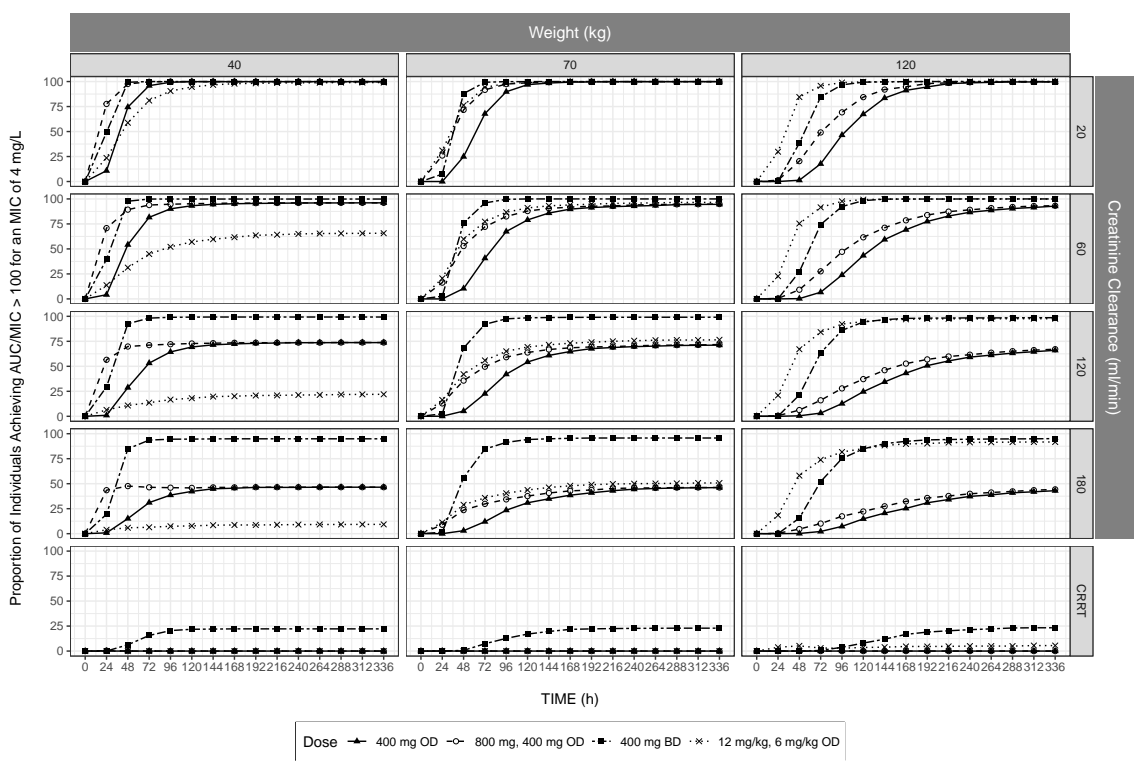

